\title{
Nassehi, Armin: Muster. Theorie der digitalen Gesellschaft.
}

\author{
München: C.H. Beck 2019. 326 Seiten. Preis: $€ 26,00$
}

\author{
Klaus Beck
}

Angenommen: 9. Dezember 2020 / Online publiziert: 18. Dezember 2020

(C) Der/die Autor(en) 2020

Systemtheoretiker sind keine Systemkritiker. Sie zeichnet die eher leidenschaftslos nüchterne Beobachtung von Funktionen und Strukturen aus. Dieser Perspektive folgt der Münchener Soziologe Armin Nassehi, der entgegen des Trends keinen Beitrag dazu leisten möchte, die möglicherweise schädlichen sozialen, kulturellen, politischen Folgen oder ethischen Probleme dessen zu kritisieren, was landauf landab mitunter alarmistisch als Digitalisierung diskutiert wird. Es geht nicht um Überwachung, Datenschutz und Freiheitsrechte. Nassehi möchte wissen, welches Problem Digitalisierung löst. Im Grunde kennt der Systemtheoretiker die Antwort schon, und Nassehis Duktus im gesamten Buch lässt keine Zweifel daran, dass er persönlich schon besonders früh erkannt hat: Komplexität bzw. die Reduktion von Komplexität wird durch eine digitale Beobachtung der Gesellschaft gelöst, die Regelmäßigkeiten oder eben die titelgebenden „Muster“ erkennt. Digitalisierung, so Nassehi, ist ein gesellschaftlicher Prozess, der die Frage aufwirft, ,,was an der gesellschaftlichen Moderne womöglich vorher schon ,digital " war" (S. 17) und damit der gegenwärtigen Digitalisierung erst den Boden bereitet hat. Digitalisierung ist aus seiner Sicht nicht nur ein soziales, sondern geradezu ein soziologisches Projekt, und so macht er sich auf die Suche nach der irgendwie immer schon digitalen Struktur der Gesellschaft.

Je weicher die Definitionen, umso mehr fällt unter den Begriff: Wenn alles, was mit Zahlen (Daten) und Zählen verbunden ist, digital ist, dann fängt die Digitalisierung der Gesellschaft oder eben die digitale Gesellschaft tatsächlich mit der öffentlichen Sozialstatistik an (S. 31). Was man gut 100 Seiten weiter liest, klingt dann allerdings wie ein Dementi, denn schlichte Zählbarkeit dürfe nicht als Digitalisierung missverstanden werden (S. 146). Die zählende Gesellschaft beobachtet

Prof. Dr. K. Beck ( $₫)$

Lehrstuhl für Kommunikationswissenschaft, Universität Greifswald,

Ernst-Lohmeyer-Platz 3, 17489 Greifswald, Deutschland

E-Mail: klaus.beck@uni-greifswald.de 
sich selbst als (soziale) Welt und verdoppelt diese dabei in Datenform, die Muster aufzeigt. Fünfzig Seiten später entdeckt Nassehi, dass auch die Schrift die Welt verdoppelt. Für die Semiotik bahnt sich damit keine Sensation an, nur dass sie die Zeichenbeziehungen komplexer als Dreieck modelliert, und damit dem Beobachter die Aufmerksamkeit schenkt, die er auch bei der digitalen Mustererkennung verdienen würde.

Muster schaffen (wie Erzählungen übrigens auch) Ordnung, reduzieren die Komplexität des modernen Zusammenlebens, die Ulrich Becks Risikogesellschaft und Pierre Bourdieus Distinktionstheorie umreißen (Nassehi greift kurz darauf zurück, ohne die Kollegen zu erwähnen). Die empirische Sozialforschung quantitativer wie qualitativer Provenienz versucht, durch Beobachtung Regelmäßigkeiten und Muster aufzuweisen, soziale Ordnung zu schaffen, nicht etwa diese Ordnungen auf ihre Kontingenzen zu hinterfragen. Andererseits ,,verändert“ die Digitaltechnik ,,zunächst nicht die Gesellschaft, sondern die Gesellschaft erfordert es geradezu“ (S. 56), dass sich Entscheider und Verwalter der digitalen Muster bedienen. Erstaunlicherweise spielt der Beobachter der Muster erneut keine Rolle. Aber stecken die Muster in den Daten oder gar in der Gesellschaft? Und müsste man aus sozial- wie aus medienwissenschaftlicher Sicht nicht auch analysieren, wer da eigentlich beobachtet, welche korporativen Akteure mit welchen Interessen wen beobachten?

In der von Nassehi völlig ignorierten kommunikationswissenschaftlichen Forschung, aber auch in der techniksoziologischen gibt es hier durchaus Überlegungen, wie Affordanzen und Restriktionen die Beobachtung durch Algorithmen strukturieren. Für Nassehi scheint es keinen Algorithmic Bias zu geben, denn der „Algorithmus berechnet nur ein Muster, unabhängig von Sinn“ (S. 75). Knapp zehn Seiten widmet Nassehi der „Digitalisierung der Kommunikation“ und es ist schon beeindruckend, wie souverän hier der Forschungsstand ignoriert und wie wenig die Grundbegriffe Information, Medium oder Kommunikation reflektiert werden.

Im Zuge der Argumentation entpuppt sich die Digitalisierung der vermeintlich immer schon digitalen Moderne dann doch als epochales Problem, ja als ,ähnliche Katastrophe wie der Buchdruck für den Informationshaushalt der Gesellschaft" (S. 119) und nun werden die Folgeprobleme, die doch keine Rolle spielen sollten, nacherzählt. Wo überhaupt eine Auseinandersetzung mit diesen „Störungen“ stattfindet, überzeugt sie mich nicht: Die „,bürgerliche Buchwelt“, das ,,pädagogisch motivierte" (S. 137) Tagebuchschreiben und -lesen unterscheidet sich von den derzeit propagierten Selftracking- und Selbstoptimierungsangeboten grundlegend im Hinblick auf die (von Nassehi negierte) informationelle Selbstbestimmung und die bürgerliche Autonomie. ,Solche Daten fallen schlicht an“- meint Nassehi mit Blick auf sein Smartphone (S. 147) und die „Selbstpraktiken der Digitalwelt kommen ganz ohne Motive ... und große Erklärungen“ aus. Das deckt sich nicht wirklich mit dem Forschungsstand, und als Kommunikations- und Medienforscher dürfen wir das wohl anders sehen.

Nassehi will eine Strukturähnlichkeit von differenzierten modernen Gesellschaften und binär codierter Digitaltechnik erkennen, doch am Ende dieser Analyse steht nicht mehr als die Binsenweisheit, dass digitale Computertechnik nahezu universell einsetzbar ist. Wo die sozialen Grenzen und Risiken dieser Annahme liegen, hat Evgeny Morozov mit dem Begriff des „Solutionism“ klarer erkannt. 
Zirkulär mutet die Begründung der ,strukturellen Digitalität der modernen Gesellschaft" an: Luhmanns empirisch unhinterfragte binäre Codierung der gesellschaftlichen Funktionssysteme wird nicht als Setzung oder Beobachtung, sondern als Beleg für die Ähnlichkeit zu digitaler Technik herangezogen (S. 171). Und dabei handele es sich ausdrücklich nicht um Luhmann'sche Metaphorik, sondern es verhält sich „tatsächlich“ so (S. 171-175). Essentialisierungen finden sich auch bei der ,Digitalität des Digitalen“ oder wenn der Kontrollüberschuss der Datenwelt sich „,kaum kontrollieren“ lässt, weil er ,gewissermaßen in der Natur der Sache“ liegt (S. 181). Plausibel hingegen ist, dass Digitalisierung nicht als neues Funktionssystem, Fremdkörper (S. 177) oder Ende einer Epoche (S. 184) betrachtet wird, was wir allerdings seit der Analyse soziotechnischer Systeme bereits wissen.

Die Kapitelüberschrift „Das Internet als Massenmedium“ dürfte in unserem Fach kaum noch als Titel einer (bestandenen) Hausarbeit durchgehen, doch für Nassehi ist das „Internet selbstverständlich ein Massenmedium, weil es tatsächlich weltumspannend ist“ (S. 263). Alternativ dazu werden die „Massen von Information“ (S. 286; offenbar als Substanzbegriff gedacht) als Begründung herangezogen. Ein paar Seiten weiter werden nicht nur die Gefährdung, sondern sogar die Möglichkeit der informationellen Selbstbestimmung negiert, weil Information jetzt als „Beobachtungskorrelat" gilt, das gar nicht selbstbestimmt abgegeben werden kann. Auch hier wäre ein Blick auf semiotischen Zeichentypen und die Intentionalität von Kommunikation hilfreich gewesen. Aus der Liste populärer Irrtümer ruft Nassehi auch die Mär von der einseitigen Massenkommunikation zwischen Sender und Empfänger sowie der Umkehr der Verhältnisse im Netz auf. Medialität entsteht demnach, wenn Kommunikation zwischen Sender und Empfänger ,,von Dritten beobachtet werden kann“" (S. 281). Jahrzehnte des kommunikations- und medienwissenschaftlichen Forschungsdiskurses, Dutzende von Lehrbüchern und Lexika, Lösungsangebote wie Hybridmedium, die Unterscheidung von Medien erster und zweiter Ordnung, Medienwandel - nichts davon interessiert hier. Dass diese Überreduktion von Komplexität zur Stabilität seines Theoriesystems beiträgt, darf man bezweifeln. Rezipiert werden hingegen Bestseller wie Zuboffs Überwachungskapitalismus, nur dass Nassehi hier kein Geschäftsmodell, ja nicht einmal eine „Intention“ (S. 268) erkennen mag. Fest steht ohnehin: „Das Problem ist nicht der Kapitalismus“ (S. 323). Der Unterschied zur staatlichen Überwachung und Disziplinierung, die seit Jahrhunderten auch analog stattfindet, verschwimmt - und die Abwehr staatlicher Regulierung wird mit der Oberflächlichkeit der Kritik an Big Data und der Naivität des Glaubens an staatliche Maßnahmen begründet.

Die Überbetonung von Kontinuität der ,Digitalisierung der bereits digitalen Gesellschaft" (S. 263) erscheint unter dem Strich genauso wenig hilfreich, wie das Ausrufen einer digitalen Revolution, neuer Epochen oder Bindestrich-Gesellschaften.

Funding Open Access funding enabled and organized by Projekt DEAL.

Open Access Dieser Artikel wird unter der Creative Commons Namensnennung 4.0 International Lizenz veröffentlicht, welche die Nutzung, Vervielfältigung, Bearbeitung, Verbreitung und Wiedergabe in jeglichem Medium und Format erlaubt, sofern Sie den/die ursprünglichen Autor(en) und die Quelle ord- 
nungsgemäß nennen, einen Link zur Creative Commons Lizenz beifügen und angeben, ob Änderungen vorgenommen wurden.

Die in diesem Artikel enthaltenen Bilder und sonstiges Drittmaterial unterliegen ebenfalls der genannten Creative Commons Lizenz, sofern sich aus der Abbildungslegende nichts anderes ergibt. Sofern das betreffende Material nicht unter der genannten Creative Commons Lizenz steht und die betreffende Handlung nicht nach gesetzlichen Vorschriften erlaubt ist, ist für die oben aufgeführten Weiterverwendungen des Materials die Einwilligung des jeweiligen Rechteinhabers einzuholen.

Weitere Details zur Lizenz entnehmen Sie bitte der Lizenzinformation auf http://creativecommons.org/ licenses/by/4.0/deed.de.

Prof. Dr. Klaus Beck ist Inhaber des Lehrstuhls für Kommunikationswissenschaft der Universität Greifswald. 